\title{
Inertia Compensation While Scanning Screw Threads on Coordinate Measuring Machines
}

\author{
Sergey Kosarevsky ${ }^{1 *}$ and Viktor Latypov ${ }^{2}$ \\ ${ }^{1}$ Faculty of Technology, Saint-Petersburg Institute of Machine-Building, Saint-Petersburg, Russia 195197 \\ ${ }^{2}$ Faculty of Applied Mathematics and Control Processes, Saint-Petersburg State University, Saint-Petersburg, Russia 198504 \\ Usage of scanning coordinate-measuring machines for inspection of screw threads has become a common practice \\ nowadays. Compared to touch trigger probing, scanning capabilities allow to speed up the measuring process while still \\ maintaining high accuracy. However, in some cases accuracy drastically depends on the scanning speed. In this paper \\ a compensation method is proposed allowing to reduce the influence of inertia of the probing system while scanning \\ screw threads on coordinate-measuring machines.
}

Keywords: Scanning, CMM, accuracy, screw thread.

\section{INTRODUCTION}

$\mathrm{N}^{\mathrm{v}}$ UMERICAL COMPENSATION of measurement errors is a well developed field of metrology [4]. This type of compensation originates from different CAA (computer-aided accuracy) methods and is extensively used in modern CMMs and CNC machines during recent years [12], [8].

To successfully compensate measurement results one should clearly understand the exact source of errors. Measuring uncertainty on coordinate-measuring machines depends on many factors. They can be summarized into the following types [10]:

- kinematic;

- thermo-mechanical;

- loads;

- dynamic;

- control software.

Some dynamic effects produce non-constant measurement errors varying according to known models. These models depend entirely upon specific measurement tasks and development of a generic model is troublesome. One of the widely accepted examples of such kind of model is pretravel compensation [5], [13], [14].

In this paper a novel compensation method is given to reduce minor and major diameter measurement errors from inertia effects emerging while scanning screw threads.

\section{RELATED WORK}

Due to inertial forces during high-speed scanning of freeform workpieces with low radius of curvature on coordinatemeasuring machines it is difficult for the control software to maintain stable contact between the probe and the surface. This is one of the reasons preventing accurate scanning

\footnotetext{
*Corresponding author: kosarevsky@linderdaum.com
}

at high speeds [9]. The study of dynamic effects was conducted by many researchers. Application of signal analysis and processing theory to dynamic metrology was studied in [7]. Pereira and Hocken [9] proposed classification and compensation methods for dynamic errors of scanning coordinatemeasuring machines. They used Taylor series and Fourier analysis to compensate measurement of circular features. ISO 10360 [1] defines acceptance tests for scanning coordinatemeasuring machines. Farooqui and Morse [6] proposed reference artifacts and tests to compare scanning performance of different coordinate-measuring machines. Szelewski et al. [11] conducted experimental research and concluded that free-form surfaces scanning time reduction is limited at high speeds by acceleration and deceleration of the probing system.

\section{Theoretical Model of Probe Inertial Motion}

High-speed longitudinal scanning of a thread surface results in loss of contact between the probe and the thread surface due to inertial forces (Fig. 1, thread axis goes vertically). Because of this, the coordinate-measuring machine will measure points not belonging to the thread surface. The actual value of the minor diameter (for internal threads) or the major diameter (for external threads) can be distorted. This effect is noticeable at scanning speeds above $5 \mathrm{~mm} / \mathrm{s}$ and the probing system mass of over $0.1 \mathrm{~kg}$.

The "helix" scanning strategy is free from this effect since no surface part with low radius of curvature is scanned at high speed.

Energy conservation yields (Fig. 1):

$$
\frac{m \cdot\left(V_{\text {scan }} \cdot \vec{N}\right)^{2}}{2}=|\vec{F}| \cdot \delta,
$$

where $m$ is the mass of the probing system floating inside the CMM probing head, $\mathrm{kg} ; V_{\text {scan }}$ is the scanning speed, $\mathrm{m} / \mathrm{s} ; \vec{F}$ 


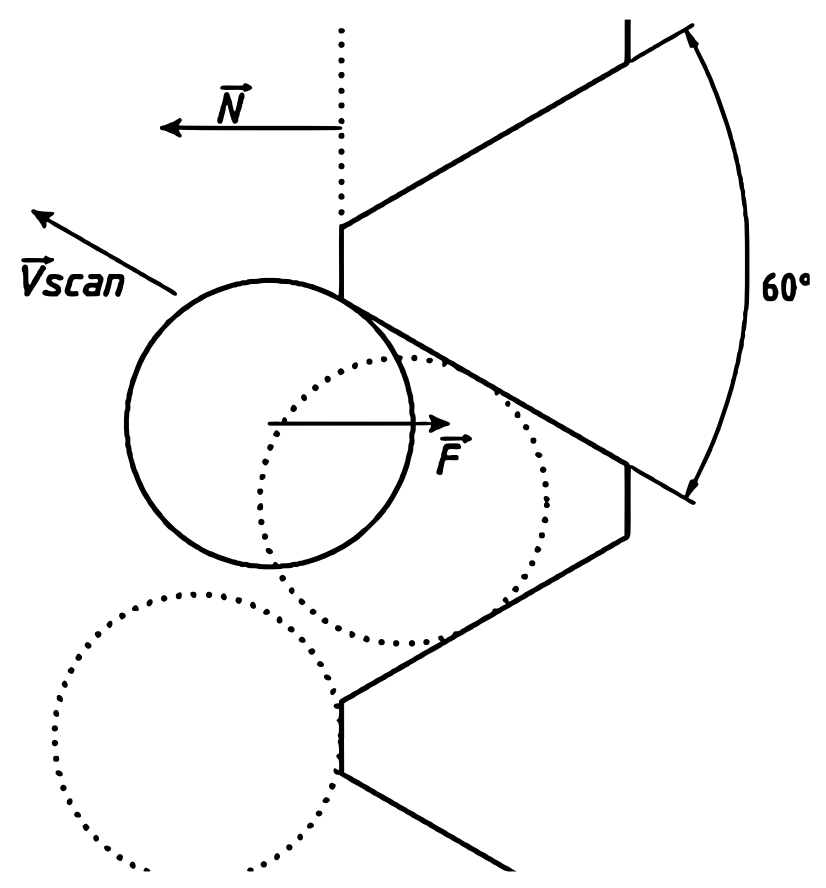

Fig. 1: Scanning thread surface with a spherical probe.

is the contact force, $\mathrm{N} ; \vec{N}$ is the unit vector orthogonal to the thread axis; $\delta$ is the separation of the probe from the surface (Fig. 2), m.

Assuming that $\vec{N}$ and $\vec{F}$ are collinear, the separation value $\delta$ can be derived from the equation (1):

$$
\delta=\frac{m \cdot V_{\text {scan }}^{2} \cdot \cos ^{2} \frac{\alpha}{2}}{2 \cdot F},
$$

where $\alpha$ is the thread angle.

Since the separation $\delta$ is axisymmetric (Fig. 2), the diameter measurement error is

$$
D_{\delta}=\frac{m \cdot V_{\text {scan }}^{2} \cdot \cos ^{2} \frac{\alpha}{2}}{F} .
$$

\section{COMPENSATION}

The equation (2) can be used to obtain the diameter compensation formula:

$$
D=D_{m} \pm \frac{m \cdot V_{\text {scan }}^{2} \cdot \cos ^{2} \frac{\alpha}{2}}{F},
$$

where $D$ is the compensated diameter, m (Fig. 2); $D_{m}$ is the measured diameter, $m$. The upper sign applies to internal threads and the lower sign to external threads, respectively.

\section{EXPERIMENTAL VERIFICATION OF THE MODEL}

We scanned the ISO metric screw thread ring gauge M170x6 [3] to measure its minor diameter. The diameter was calculated as the maximal inscribed cylinder with both filtering and

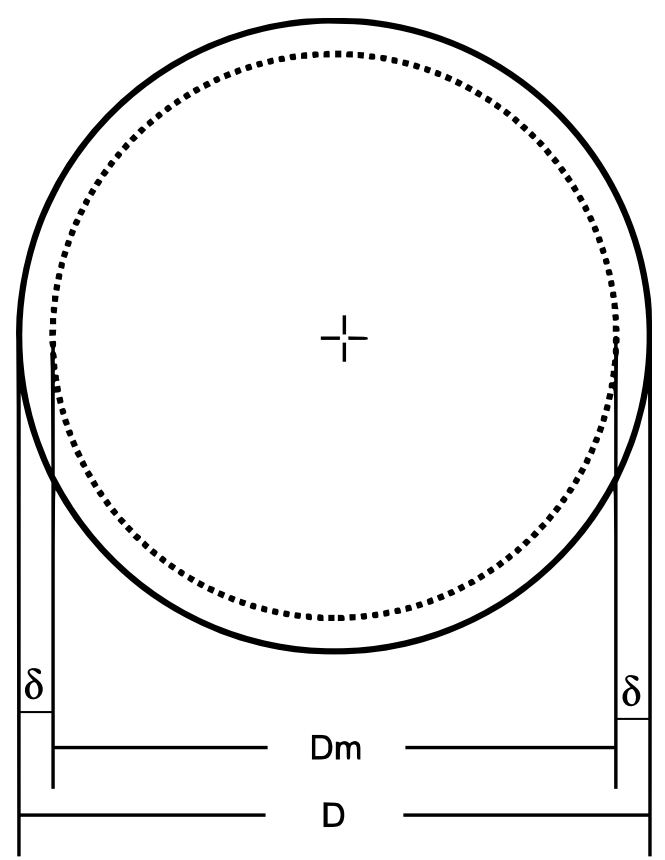

Fig. 2: Compensated and measured diameters.

outliers elimination disabled. The mass of our probing system was $0.1 \mathrm{~kg}$ according to manufacturer's specification, contact force was set in CMM software to $0.2 \mathrm{~N}$.

Theoretical and experimental values for different scanning speeds are summarized in Table 1.

Experimental values were obtained on a Carl Zeiss PRISMO 10 S-ACC coordinate-measuring machine with the following parameters according to ISO 10360 [1]:

- maximum permissible indication error

$$
M P E_{E}=1.7+\frac{L}{350} \mu m
$$

- maximum permissible probing error

$$
M P E_{P}=1.5 \mu m
$$

- maximum permissible scanning error

$$
M P E_{T H P}=2.1 \mu \mathrm{m} \text {. }
$$

The probing system used consists of one vertical ruby sphere stylus with the shaft length of $60 \mathrm{~mm}$ and sphere radius of $3 \mathrm{~mm}$.

The value of the minor diameter scanned at $1 \mathrm{~mm} / \mathrm{s}$ was assumed to be the actual value $\left(D_{\delta \text {.Exp. }}=0\right)$. Each of the resulted experimental errors were averaged by 10 measurements.

Plots of the estimated error $\left(D_{\delta}\right)$, the experimental error $\left(D_{\delta \text {.Exp. }}\right)$ and the compensated error $\left(D_{\delta \text {.Exp. }}-D_{\delta}\right)$ are displayed in Fig. 3. The compensated error is also individually shown in Fig. 4. 
Table 1: Effect of scanning speed on measurement results.

\begin{tabular}{ccccc}
\hline Speed, $\mathrm{mm} / \mathrm{s}$ & Separation $\delta, \mu m$ & $D_{\delta}, \mu m$ & $D_{\text {S.Exp. }} \mu m$ & Reduction, \% \\
\hline 1 & 0.2 & 0.4 & 0.0 & 0.0 \\
2 & 0.8 & 1.5 & 0.4 & 26.7 \\
3 & 1.7 & 3.4 & 1.8 & 53.0 \\
4 & 3.0 & 6.0 & 2.0 & 33.3 \\
5 & 4.7 & 9.4 & 3.1 & 33.0 \\
6 & 6.8 & 13.5 & 11.0 & 81.5 \\
7 & 9.2 & 18.4 & 11.9 & 64.7 \\
8 & 12.0 & 24.0 & 31.6 & 68.3 \\
9 & 15.2 & 30.4 & 37.6 & 76.3 \\
10 & 18.8 & 37.5 & 46.1 & 87.1 \\
11 & 22.7 & 45.4 & 38.5 & 75.2 \\
12 & 27.0 & 54.0 & 40.6 & 73.5 \\
13 & 31.7 & 63.4 & 80.2 & 76.5 \\
14 & 36.8 & 73.5 & 90.8 & 53.4 \\
\hline
\end{tabular}

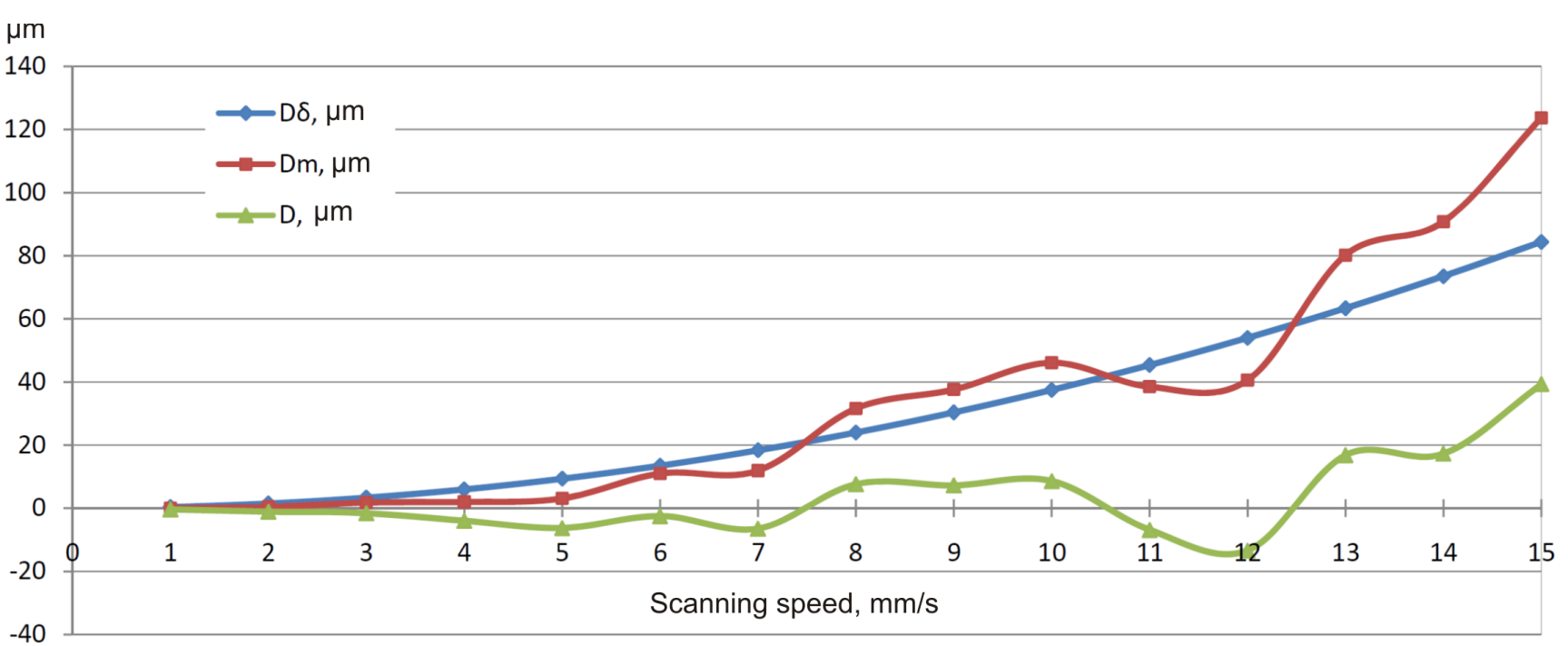

Fig. 3: Measurement error versus scanning speed.

The percentage of error reduction was calculated as

$$
R=\left(1-\frac{\left|D_{\delta . \operatorname{Exp} .}\right|-D_{\delta}}{D_{\delta}}\right) \cdot 100 \%,
$$

and is displayed in Fig. 4.

\section{CONCLUSIONS}

The most significant error reduction (Fig. 4) can be achieved in the interval of scanning speeds from $6 \mathrm{~mm} / \mathrm{s}$ and up to $12 \mathrm{~mm} / \mathrm{s}$. At scanning speeds below $5 \mathrm{~mm} / \mathrm{s}$ the influence of the studied effect is surpassed by the $M P E_{E}$ value of the tested CMM and cannot be treated unambiguously (note large fluctuations in error reduction at speeds below $5 \mathrm{~mm} / \mathrm{s}$ ). At speeds above $12 \mathrm{~mm} / \mathrm{s}$ the compensated error quickly surpasses $20 \mu \mathrm{m}$ and becomes unacceptable for most practical applications.

Even though the mass of the probing system was taken directly from the manufacturer's specification and no actual mass measurement was performed, the calculated compensation values (3) have proven to be useful.

Though numerical compensation of the measurement re- 


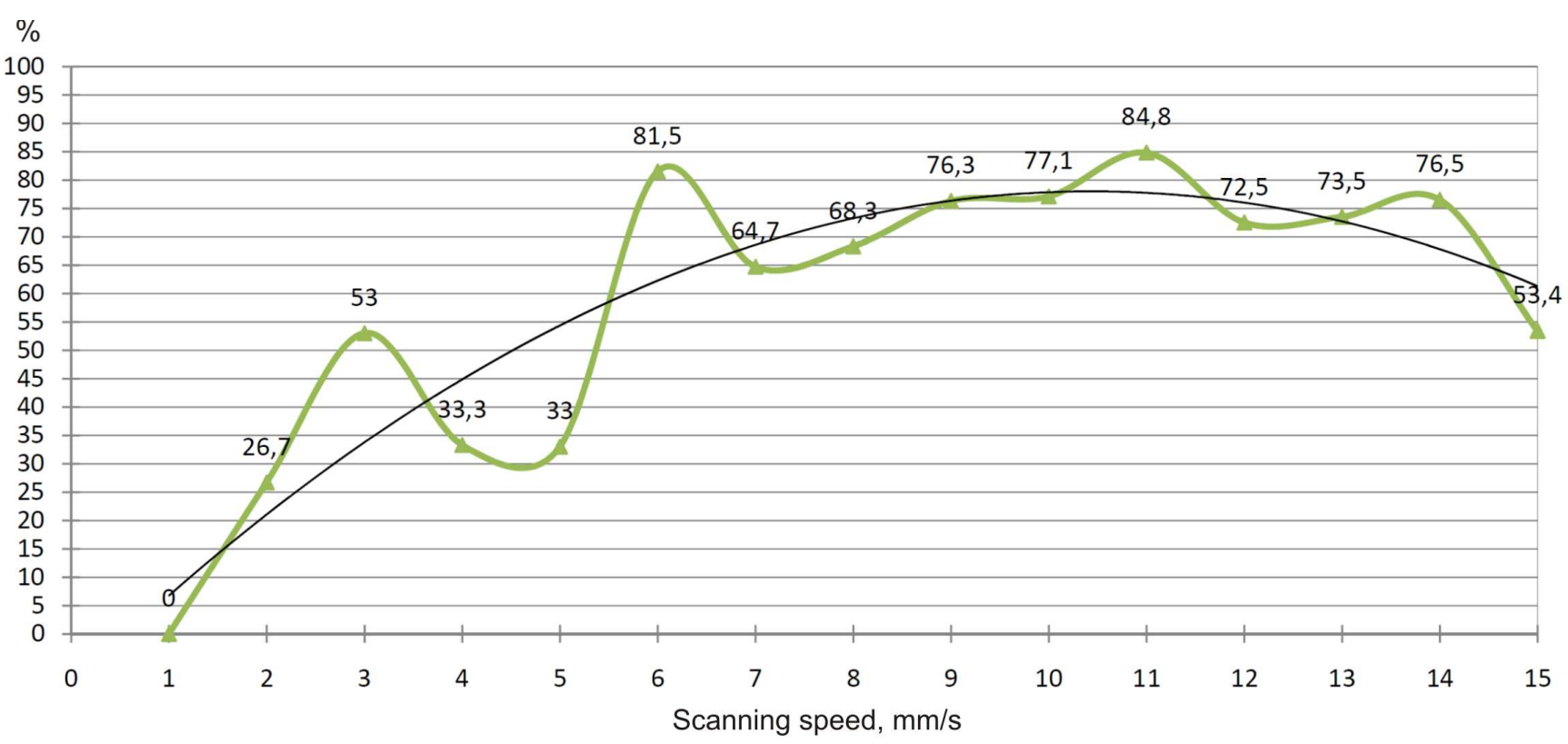

Fig. 4: Error reduction.

sults is a rapidly growing field of dimensional metrology, its application to dynamic effects compensation is to be extended. Experimental activities described in this paper confirm that the method proposed is able to reduce the influence of inertia of the probing system on measurement error of screw thread's minor and major diameters. This method can be easily implemented directly in CMM software to allow automatic inertia compensation.

\section{ACKNOWLEDGMENT}

This work was carried out with the support of the OPTEC Carl Zeiss grant 2010, http: / / optec. zeiss.ru.

\section{REFERENCES}

[1] ISO 10360-4 (2000). Geometrical Product Specifications (GPS) - Acceptance And Reverification Tests for Coordinate Measuring Machines (CMM) - Part 4: CMMs Used in Scanning Measuring Mode.

[2] ISO 11562 (1996). Geometrical Product Specifications (GPS) - Surface Texture: Profile Method - Metrological Characteristics of Phase Correct Filters. Geneva, Switzerland: International Organization for Standardization.

[3] ISO 68-1. (1998). ISO General Purpose Screw Threads - Basic Profile - Metric Screw Threads. Geneva, Switzerland: International Organization for Standardization.

[4] ISO GUM (1995). Guide to the Expression of Uncertainty in Measurement. BIPM, IEC, IFCC, ISO, IUPAC and OIML. Second Edition.

[5] Estler, W.T., Phillips, S.D., Borchardt, B., Hopp, T., Witzgall, C., Levenson, M., Eberhardt, K., McClain, M., Shen, Y., Zhang, X. (1996). Error compensation for CMM touch trigger probes. Precision Engineering, 19(2), 85-97.
[6] Farooqui, A., Morse P. (2007). Methods and artifacts for comparison of scanning CMM performance. Transactions of the ASME, 7, 72-80.

[7] Hessling, J.P. (2008). Dynamic metrology — an approach to dynamic evaluation of linear time-invariant measurement systems. Measurement Science and Technology, 19, 084008 (7pp).

[8] Jenq-Shyong, C. (1995). Computer-aided accuracy enhancement for multi-axis CNC machine tool. International Journal of Machine Tools and Manufacture, 35(4), 593-605.

[9] Pereira, P.H., Hocken, R.J. (2007). Characterization and compensation of dynamic errors of a scanning coordinate measuring machine. Precision Engineering, 31(1), 22-32.

[10] Schwenke, H., Knapp, W., Haitjema, H., Weckenmann, A., Schmitt, R., Delbressine, F. (2008). Geometric error measurement and compensation of machines - An update. CIRP Annals - Manufacturing Technology, 57, 660-675.

[11] Szelewski, M., Grzelka, M., Barisic, B. (2007). Free surface scanning with CMM and its reproduction in CAD system. Engineering Review, 27(1), 7-12.

[12] Trapet, E., Wäldele, F. (1997). Coordinate metrology — Flexibility in conflict with accuracy? Anais - Seminário Internacional de Metrologia, Florianopolis - SC.

[13] Woźniak, A., Dobosz, M. (2003). Metrological feasibilities of CMM touch trigger probes. Part I: 3D theoretical model of probe pretravel. Measurement, 34(4), 273-286.

[14] Woźniak, A., Dobosz, M. (2003). Metrological feasibilities of CMM touch trigger probes. Part II: Experimental verification of the 3D theoretical model of probe pretravel. Measurement, 34(4), 287-299.

Received December 21, 2009. Accepted March 22, 2010. 